\begin{tabular}{|l|l|}
\hline POS0746 & THE ARTHRITIS COMPONENT OF THE SLEDAI \\
SHOULD ONLY BE SCORED IF THERE IS JOINT \\
SWELLING
\end{tabular}

K. Mahmoud ${ }^{1,2}$, A. Zayat ${ }^{3}$, M. Y. MD Yusof ${ }^{1,2}$, K. Dutton ${ }^{1,2}$, L. S. Teh ${ }^{4}$, C. S. Yee ${ }^{5}$, D. D'cruz ${ }^{6}, \mathrm{~N} . \mathrm{Ng}^{6}$, D. Isenberg ${ }^{7}$, C. Ciurtin ${ }^{8}$, P. G. Conaghan ${ }^{1,2}$, P. Emery ${ }^{1,2}$, C. J. Edwards ${ }^{9}$, E. Hensor ${ }^{1,2}$, E. Vital ${ }^{1,2} .{ }^{1}$ NIHR Leeds Biomedical Research Centre, Rheumatology, Leeds, United Kingdom; ${ }^{2}$ Leeds Institute of Rheumatic and Musculoskeletal Medicine, Rheumatology, Leeds, United Kingdom; ${ }^{3}$ Bradford Teaching Hospitals NHS Foundation Trust, Rheumatology, Bradford, United Kingdom; ${ }^{4}$ Royal Blackburn Hospital, Rheumatology, Blackburn, United Kingdom; ${ }^{5}$ Doncaster \& Bassetlaw Hospitals NHS Trust, Rheumatology, Doncaster, United Kingdom; ' Guy's and St Thomas' Hospital, Rheumatology, London, United Kingdom; ${ }^{7}$ University College Hospital, Rheumatology, London, United Kingdom; ${ }^{8}$ Centre for Adolescent Rheumatology, Rheumatology, London, United Kingdom; ${ }^{9}$ University Hospital Southampton NHS Foundation Trust, Rheumatology, Southampton, United Kingdom

Background: SLE disease activity tools do not optimally define disease activity and response. The SLEDAl arthritis item is common, and sufficient to define SRI response. Lupus patients with arthralgia often have no swelling. Glossary definitions of arthritis in different versions of the SLEDAI have included: swelling, swelling between visits, effusion, tenderness, warmth and erythema. MSK ultrasound in SLE can identify synovitis without swelling, ultrasound synovitis is associated with worse symptoms and serology, predicts response to therapy, and is more responsive to therapy than clinical variables.

Objectives: To validate different glossary definitions for SLEDAI arthritis using musculoskeletal ultrasound.

Methods: We analysed baseline data from a multicentre longitudinal study. Physicians scored SLEDAI-2K in 133 patients with joint pain that was considered inflammatory, but not necessarily swelling. Stable immunosuppressants and prednisolone $<=5 \mathrm{mg}$ were permitted. If the arthritis criterion was scored, we asked physicians to report which glossary features drove their decision. Musculoskeletal ultrasound of hands and wrists was performed on the same day, blinded to clinical findings. We defined abnormal grey-scale in joints as 1 joint with GS $>=2$, and in tendons as $>=1$ tendon with $G S>=1$, and abnormal power Doppler as $>=1$ joint or tendon with PD $>=1$.

Results: 78/133 patients had arthritis scored on SLEDAI-2K. In 21/78, swelling was not a reason for that decision. These 21 patients had either tenderness (16/21), swelling reported between visits (4/21) or both of these (1/21). No patient was scored for warmth, erythema or effusion alone. Comparison of SLEDAI definitions and ultrasound is shown in Table 1. Of 57 patients with SLEDAI arthritis scored due to swelling, $90 \%$ had an abnormal ultrasound. The positive predictive value was $89 \%(95 \% \mathrm{Cl} 79-94)$. Of 21 patients with SLEDAI arthritis scored without swelling, $48 \%$ had an abnormal ultrasound. The positive predictive value was $48 \%(95 \% \mathrm{Cl} 31-67)$. There was no substantive difference in clinical and serological variables comparing patients with SLEDAI arthritis without swelling and patients without SLEDAI arthritis. In contrast, patients with SLEDAI arthritis with swelling had worse ESR $(p=0.0003)$, Physician MSK disease activity VAS $(p<0.001)$ and patient EMS VAS $(p=0.0019)$ and $\lg G(p=0.0625)$ compared to the other two groups.

Conclusion: Although ultrasound proven synovitis in the absence of swelling is not uncommon, it is not reliably identified using other signs or symptoms. The arthritis item of the SLEDAI was likely to be associated with ultrasound synovitis if scored because of swelling, but not if scored because of tenderness or swelling between visits. Our results support raising the threshold criteria for arthritis so that it should only be scored when there is joint swelling. Previous clinical trial datasets could be re-analysed excluding SLEDAI arthritis scores not confirmed by a swollen joint count greater than one.

Disclosure of Interests: Khaled Mahmoud: None declared, Ahmed Zayat: None declared, Md Yuzaiful Md Yusof: None declared, Katherine Dutton: None declared, Lee-Suan Teh: None declared, Chee-Seng Yee: None declared, David d'cruz: None declared, Nora Ng: None declared, David Isenberg: None declared, Coziana Ciurtin: None declared, Philip G Conaghan: None declared, Paul Emery: None declared, Christopher John Edwards Shareholder of: Research grant support from; Abbvie, Biogen, Pfizer, Consultant of: Personal fee from; Abbvie, BMS, Biogen, Celgene, Celltrion, Fresenius, Gilead, GSK, Janssen, Lilly, Mundipharma, Pfizer, MSD, Novartis, Roche, Samsung, Sanofi, UCB, Grant/research support from: Research grant support from; Abbvie, Biogen, Pfizer, Elizabeth Hensor: None declared, Edward Vital Speakers bureau: AstraZeneca, Genentech, Aurinia, Lilly, Modus, Consultant of: AstraZeneca, Genentech, Aurinia, Lilly, Modus, Grant/research support from: Sandoz, AstraZeneca, DOI: 10.1136/annrheumdis-2021-eular.2199

\section{POS0747 MAPPING THE NATIONWIDE CLINICAL PROFILE AND PATTERNS OF CARE OF SLE IN BRAZIL - FINDINGS FROM THE MACUNAÍMA STUDY}

M. Abreu ${ }^{1,2}$, O. Monticielo ${ }^{3}$, V. Fernandes ${ }^{4}$, A. Cristovão Maiorano ${ }^{2}$, F. Dos Santos Beserra ${ }^{2}$, F. Lamarao ${ }^{5}$, N. David ${ }^{5}$, B. De Veras ${ }^{5}$, B. Bica ${ }^{1}$, D. Sávio Nunes de Lima ${ }^{6}$, M. Maria Das Chagas Medeiros ${ }^{7} .{ }^{1}$ Universidade Federal do Rio de Janeiro, Faculdade de Medicina, Rio de Janeiro, Brazil; ${ }^{2}$ Universidade Federal do Rio de Janeiro, Laboratório para Pesquisa em Desfechos Centrados no Paciente, Rio de Janeiro, Brazil; ${ }^{3}$ Universidade Federal do Rio Grande do Sul, Serviço de Reumatologia do Hospital de Clínicas de Porto Alegre, Porto Alegre, Brazil; ${ }^{4}$ Universidade de Cuiabá, Department of Medicine, Cuiabá, Brazil; ${ }^{5}$ GlaxoSmithKline, Medical Department, Rio de Janeiro, Brazil; ${ }^{6}$ Universidade Federal do Amazonas, Serviço de Reumatologia do Hospital Universitário Getúlio Vargas, Manaus, Brazil; ${ }^{7}$ Universidade Federal do Ceará, Faculdade de Medicina, Fortaleza, Brazil

Background: Systemic lupus erythematosus (SLE) is an autoimmune disease with wide clinical variability. Brazil has vast regional diversity, both from an ethnic and socio-cultural point of view.

Objectives: To map the clinical profile of SLE in Brazil and explore how this distribution is associated with regional disparities.

Methods: This cross-sectional study (GSK Study 207353) evaluated 300 Brazilian patients $\geq 18$ years old with SLE (American College of Rheumatology [ACR] criteria, 1997) who had been under SLE care for $\geq 1$ year. Five SLE reference teaching facilities were selected, one in each of the following Brazilian regions: North (NO), Northeast (NE), Midwest (CO), Southeast (SE), and South (SU) Each region included 60 patients. Clinical and demographic characteristics, and patterns of care were measured through questionnaires completed by physicians or nurses. The SLE Disease Activity Index (SLEDAI) score described disease activity and the Systemic Lupus International Collaborating Clinics/ACR Damage Index (SDI) described damage accrual. To assess the potential association between regional disparities and clinical outcomes, a hospitalisation profile was described. A bootstrapping approach of logistic regression was used to explore potential factors associated with hospitalisation

Results: Overall, $92.3 \%$ of patients were female, with a mean (standard deviation; SD) age of 41.8 (12.7) years and a mean (SD) disease duration of 11.8 (7.9) years. Overall, $161(53.7 \%)$ patients were of Latino origin; in the NO this proportion was $88 \%$. White patients predominated in the SU $(58.3 \%)$; and black patients in the SE (31.7\%). The mean (SD) number of years of schooling was 11.3 (4.6), and was highest in the NO (14.2 [3.6]) and lowest in the SU (9.0 [4.0]; $\mathrm{p}<0.001)$. With regard to the distribution of the SLE clinical profile according to ACR criteria, arthritis was found in 221 patients and predominated in all regions (mean $73.7 \%)$, with a lower prevalence in the CO $(40 \%$; $p<0.001$; Figure $1 \mathrm{~A})$. The mean (SD) SLEDAI score was 4.33 (5.39) at the time of interview. The main contributing factors to disease activity, according to SLEDAI, were complement consumption (18\%), arthritis (15.3\%), and alopecia (15\%). The SDI scale was scored for cataracts $(15 \%)$, proteinuria $(8.7 \%)$, and thrombosis $(7.3 \%)$. Among the associated comorbidities, hypertension was predominant in the NO $(35 \%$; $\mathrm{p}=0.001)$. Smoking predominated in the SU $(23 \%$; $p<0.001)$; obesity $(27 \%$; $p=0.059)$ and dyslipidemia $(35 \% ; p=0.023)$, in the SE. Regarding patterns of care (Figure 1B), antimalarials were most frequently prescribed in the SE $(88.3 \%)$ and the SU (91.7\%). Corticosteroids prevailed in the NO (96.7\%). The mean (SD) time between home and care facility was 4.5 (12.6) hours. Patients in the NO reported the longest transport time to reach the care facility (11.5 [25.4] hours; $p<0.001)$. The hospitalisation rate during the study period was $21.3 \%$ across all regions, with no statistical difference between centres $(p=0.651)$. Reasons for

\begin{tabular}{|c|c|c|c|c|c|c|c|c|c|}
\hline & $\begin{array}{l}\text { GS joints } \\
\text { n/N (\%) }\end{array}$ & $\begin{array}{l}\text { PD joints } \\
\text { n/N (\%) }\end{array}$ & $\begin{array}{c}\text { GS tendons } \\
\text { n/N (\%) }\end{array}$ & $\begin{array}{c}\text { PD tendons } \\
\text { n/N (\%) }\end{array}$ & $\begin{array}{c}\text { Any abnormality } \\
\text { n/N (\%) }\end{array}$ & $\begin{array}{l}\text { Sensitivity } \\
\%(95 \% \mathrm{Cl})\end{array}$ & $\begin{array}{l}\text { Specificity } \\
\%(95 \% \mathrm{Cl})\end{array}$ & $\begin{array}{c}\text { PPV } \\
\%(95 \% \mathrm{Cl}) \\
\end{array}$ & $\begin{array}{c}\text { NPV } \\
\%(95 \% \mathrm{Cl}) \\
\end{array}$ \\
\hline All patients $(n=133)$ & $77 / 133(58)$ & $33 / 133(25)$ & $36 / 133(27)$ & $27 / 106(20)$ & $83 / 133(62)$ & - & - & - & - \\
\hline -Due to swelling $(n=57)$ & $48 / 57(84)$ & $28 / 57$ (49) & $21 / 57(37)$ & 19/57 (33) & $51 / 57(90)$ & $61(50,71)^{\mathrm{c}}$ & $88(75,95)^{\mathrm{c}}$ & $89(79,94)^{\mathrm{c}}$ & $57(50,64)^{\mathrm{c}}$ \\
\hline -Without swelling $(n=21)^{a}$ & $9 / 21(43)$ & $3 / 21$ (14) & $6 / 21(29)$ & $5 / 21(24)$ & $10 / 21$ (48) & $31(16,50)^{d}$ & $75(60,87)^{d}$ & $48(31,67)^{d}$ & $60(45,68)^{d}$ \\
\hline
\end{tabular}

